\title{
A numerical solver of 3D Poisson Nernst Planck equations for functional studies of ion channels
}

\author{
S. Furini ${ }^{1}$, F. Zerbetto ${ }^{2}$ \& S. Cavalcanti ${ }^{1}$ \\ ${ }^{1}$ Department of Electronics Computer Science and Systems, \\ University of Bologna, Italy \\ ${ }^{2}$ Department of Chemistry “G. Ciamician”, University of Bologna, Italy
}

\begin{abstract}
Recent results of X-Ray crystallography have provided important information for functional studies of membrane ion channels based on computer simulations. Because of the large number of atoms that constitute the channel proteins, it is prohibitive to approach functional studies using molecular dynamic methods. To overcome the current computational limit we propose a novel approach based on the Poisson, Nernst, Planck electrodiffusion theory. The proposed numerical method allows the quick computation of ion flux through the channel, starting from its 3D structure. We applied the method to the KcsA potassium channel obtaining a good accordance with the experimental data.
\end{abstract}

Keywords: membrane protein, computer simulation, ion fluxes.

\section{Introduction}

Ion channels are protein molecules embedded in the lipid bilayer of the cell membranes. They control the ion fluxes through the cellular membrane playing a central role in several cell functions, i.e. the cellular excitability [1]. In the last few years, thanks to the structural data provided by X-ray crystallography, it has become possible to analyze the channels at atomic level. In particular, the atomic structures of several bacterial ion channels selectively permeable to potassium ions - KcsA [2], MthK [3], and KvAP [4] - were revealed. The peculiar characteristic of potassium channels is the ability to conduct at a rate close to the diffusion limit $\left(10^{8}\right.$ ions/s) keeping a high selectivity $\left(\mathrm{K}^{+}\right.$is $10^{4}$ times more permeant than $\mathrm{Na}^{+}$). Since high fluxes need conduction mechanisms without 
energetic barriers, while selectivity needs a close interaction between ions and channel, these characteristics seemed incompatible. The knowledge of the KcsA atomic structure, the first potassium channel crystallized, has permitted to solve this apparent contradiction by molecular dynamic methods [5].

KcsA is made by four identical subunits, symmetrically placed around the channel axis. Each subunit consists of 160 amino acids and is characterized by three $\alpha$ helix structures - the outer helix, the pore helix and the inner helix placed like in figure 1. On the extracellular side the conduction pathway is lined by the carbonyl oxygens of the amino acid sequence TVGYG, one from each subunit. This region, named selectivity filter, is $12 \AA$ long with a mean radius of $1.4 \AA$ and it is widely conserved among different potassium channels. Below the selectivity filter the channel opens in a wide chamber with a mean radius of $6 \AA$ connected to the intracellular space by a $18 \AA$ long hydrophobic pore. Both the chamber and the hydrophobic pore are lined by the four inner helixes. Close to the intracellular mouth a bundle between these helixes reduces the hydrophobic pore radius near to $0.5 \AA$, preventing ion fluxes through the channel. Electron paramagnetic resonance experiments [6] and structural data from other potassium channels [3], suggest that KcsA opening is due to an outward movement of the inner helixes, realized by a bending of these helixes at a hinge glycine (GLY 99). The conservation of this amino acid among many potassium channels suggests a common mechanism for channel gating.

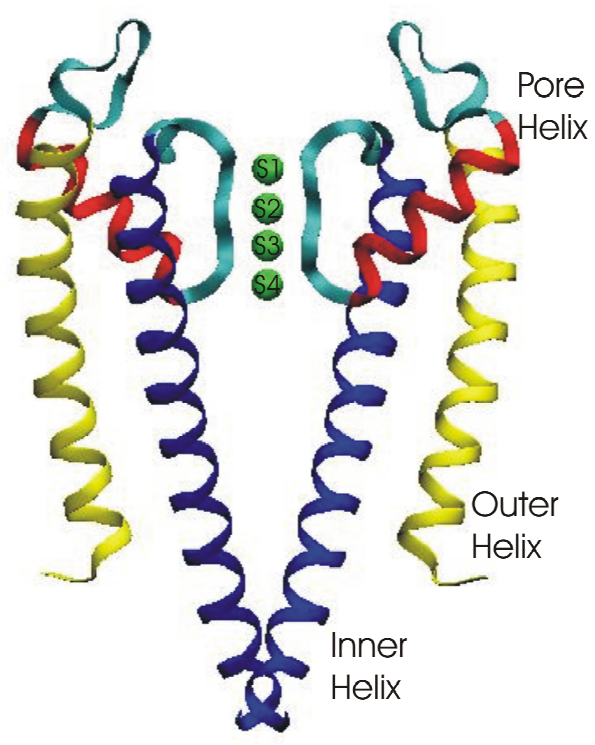

Figure 1: Two subunits of the KcsA channel. S1, S2, S3 and S4 are the four ion binding sites in the selectivity filter. 
Experimental data [2] and molecular dynamic simulations [5] have revealed the presence of four ion binding sites inside the selectivity filter, named respectively S1, S2, S3, and S4 (see Fig. 1). The energetically favoured configurations have two potassium ions in the filter, separated by a water molecule. The rapid switching between the configuration with ions in S1 and S3, and the one with ions in S2 and S4, is possible thanks to the presence of a low energetic barrier. Ion transport takes place when a third ion enters the pore from one side driving out an ion on the opposite side. Molecular dynamics simulations have showed that no energy barrier is present between the states involved in the conduction mechanism, giving an explanation for the high conduction rate [5]. The lack of energetic barriers is due to the presence of the carbonyl oxygens of the selectivity filter. These atoms mimic the presence of water hydration oxygens, cutting out the energetic cost of dehydration. The conduction mechanism works perfectly with potassium ions, but fails with smaller particles, like sodium ions. Free energy perturbation simulations have revealed that, inside the filter, the substitution of potassium by sodium is energetically expensive, with an energetic cost in line with the experimental selectivity of potassium channels [5].

Molecular dynamic simulation has pointed out the atomic details of conduction and selectivity, but is not able to reproduce the ion fluxes through the channels. Since electrical current is the main functional characteristic of ion channels, and the only one experimentally determinable, simulation of ion fluxes is the only way to link structural and functional data. The complexity of the system, due to the huge number of atoms, limits the dynamic simulations to the nanosecond scale while biological process, like ion conduction, are far slower (millisecond scale). A simplified mathematical model of ion conduction is so needed. Since most of the complexity in ion channel dynamic simulations lies in the high number of water molecule, a continuum description of the solvent is the first step to reduce the computational resources. Brownian dynamic simulations are a possible approach based on this idea. In a Brownian dynamic simulation only ions preserved their discrete nature, the solvent is described by diffusion coefficients and stochastic collisions with ions and the effect of the protein by a potential energy function. The potential energy function and the diffusion coefficients are the basic elements of this method, and to avoid arbitrariness in the mathematical model these functions are computed starting from the atomic structure of the channel. A Brownian dynamic simulation based on this approach was used by Bernechè [7] on KcsA, getting a good accordance with the experimental data.

In this study we present a further simplified approach based on the Poisson, Nernst, Planck (PNP) theory of electrodiffusion, which use a continuum description of the whole system. The next section describes the theory and the numerical algorithm developed to solve the PNP equations. The application of the method to the KcsA channel is then described. The paper concludes with a brief discussion of the results. 


\section{Theory and method}

\subsection{PNP theory}

The PNP electrodiffusion theory describes a steady state condition for a system of mobile charges. In membrane channels, the mobile charges are the different ion species in solution, which space distributions are described by the concentrations $\mathrm{C}_{\mathrm{i}}(\mathrm{r})$ (subscript $\mathrm{i}$ marks the $\mathrm{i}$-th ion specie; $\mathrm{r}$ is the position in the space). Assuming the electric field as the only driving force acting on ions, the steady state flux of the i-th ion specie has the form:

$$
J_{i}(r)=-D_{i}(r) \nabla C_{i}(r)-\mu_{i}(r) C_{i}(r) z_{i} e \nabla \psi(r)
$$

where $D_{i}(r), \mu_{i}(r), z_{i}$ are respectively diffusion coefficient, mobility and valence of the $\mathrm{i}$-th ion specie, e is the elementary charge and $\psi$ the electrostatic potential. The first term, which is proportional to the concentration gradient, is due to diffusion processes, while the last is produced by the electric field. Since the PNP theory describes a steady state condition, fluxes are time independent, and in virtue of the mass conservation law, the divergence of $\mathrm{J}_{\mathrm{i}}(\mathrm{r})$ is zero. This gives the set of differential equations:

$$
\operatorname{div}\left[D_{i}(r)\left[\nabla C_{i}(r)+\frac{e z_{i}}{k_{B} T} C_{i}(r) \nabla \psi(r)\right]\right]=0 \quad i=1, \ldots, N
$$

where $\mathrm{N}$ is the number of ion species, $\mathrm{k}_{\mathrm{B}}$ the Boltzmann's constant and $\mathrm{T}$ the absolute temperature. In (2) the Einstein's relation between diffusion coefficients and ion mobility, $\mu / \mathrm{D}=\mathrm{k}_{\mathrm{B}} \mathrm{T}$, was introduced. To complete the mathematical model it is necessary to define how electrostatic potential and ion concentrations are connected. This relation may be defined by the Poisson's equation:

$$
\operatorname{div}[\varepsilon(r) \nabla \psi(r)]=-\rho(r)-\sum_{i=1}^{N} e z_{i} C_{i}(r)
$$

where $\varepsilon(r)$ is the dielectric constant and $\rho(r)$ the charge distribution of the protein atoms that differently from ion charge distribution is assumed fixed in the space.

In the present study we included in the water solution only two monovalent ion species: one positive $(\mathrm{i}=+)$ and one negative $(\mathrm{i}=-)$. Then, the PNP differential equation set (2) and (3) reduced to:

$$
\left\{\begin{array}{l}
\operatorname{div}\left[D_{+}(r)\left[\nabla C_{+}(r)+\frac{e}{k_{B} T} C_{+}(r) \nabla \psi(r)\right]\right]=0 \\
\operatorname{div}\left[D_{-}(r)\left[\nabla C_{-}(r)-\frac{e}{k_{B} T} C_{-}(r) \nabla \psi(r)\right]\right]=0
\end{array}\right.
$$




$$
\operatorname{div}[\varepsilon(r) \nabla \psi(r)]=-\rho(r)-e\left[C_{+}(r)-C_{-}(r)\right]
$$

Once solved these equations, as described in the next section, ion concentrations and electrostatic potential in the space are obtained. Then, the ion fluxes in the channel can be computed by equation (1).

\subsection{Computational method}

The differential equations (4) and (5) were numerically solved on a cubic volume formed by $200^{3}$ cubic grid elements (the side of the grid element was $0.5 \AA$ ). The cubic volume was divided in three distinct sub-volumes: the ion channel, the membrane and the water solution (see Fig. 2). The position, the radius and the partial charge of all the atoms made up the ion channel sub-volume. Channel was discretized on the grid by using the discretization algorithm implemented in DELPHI v.4, a well-known Poisson-Boltzmann equation solver [8]. The channel was placed with its geometric centre in the centre of the cube and with the pore axis orthogonal to the upper and lower faces. The extracellular side of the channel pointed to the upper face. The height of cube was chosen double of the channel length along the pore axis ( $\mathrm{z}$ axis). To separate extra- and intra-cellular spaces outside the ion channel, a sub-volume surrounding the channel and extending between two planes orthogonal to the pore axis was considered. This volume simulated the lipid bilayer of the cell membrane. The water solution spread all over the volume not occupied by the channel and by the membrane. It was characterized by two space-dependent diffusion coefficients, one for each ionic specie. A specific dielectric constant was assigned to water solution, membrane and channel sub-volumes.

The Poisson's equation (5) was solved in the whole volume, while the mass conservation equations (4) were solved in the water solution only. As boundary condition for the Poisson's equation we assigned the electrostatic potential on the six faces of cube. The potential was set to 0 on the upper face, whereas the membrane potential value $\left(\mathrm{V}_{\mathrm{m}}\right)$ to be simulated was applied on the lower face. On the side faces a linear interpolation between 0 and $V_{m}$ was used. Two different boundary conditions were assigned for the mass conservation equations. On the upper and lower faces the boundary conditions were the ion concentrations to be simulated. On both faces anion and cation concentrations were set equal, to have electrically neutral boundaries. Boundary conditions on the side faces and at the separation surface with channel and membrane were:

$$
J_{+} \cdot n=0 \quad J_{-} \cdot n=0
$$

where $\mathrm{n}$ is the surface normal vector. In this way no ion flux was allowed through these surfaces.

Once defined the boundary conditions, the differential equation set was solved by an iterative scheme. The electrostatic potential was first computed solving the Poisson's equation with ion concentrations set to zero. This potential was used to compute ion concentrations by the solution of mass conservation equations, then the new concentrations were used to update the electrostatic 
potential. This procedure was repeated until a self-consistent solution was found. The convergence was tested by the root mean square deviation between two successive iterations. All the differential equations were solved as described in the Appendix.

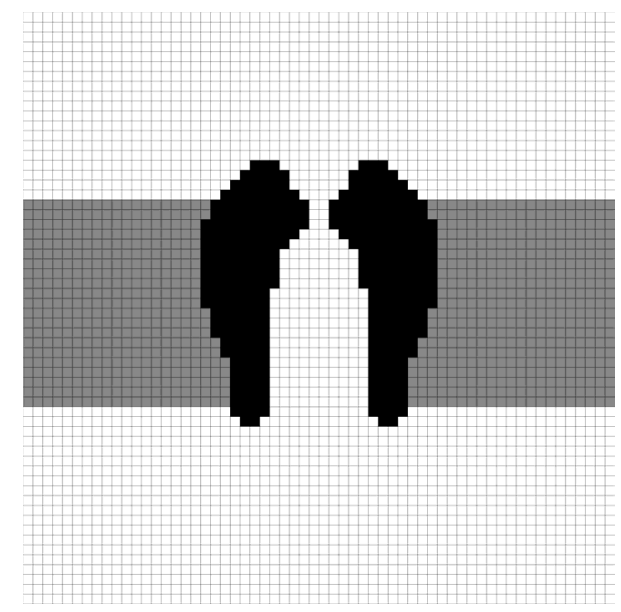

Figure 2: A 2D slice of the cubic grid. The channel is in black, the membrane in grey and the water solution in white.

\section{Application to the KesA channel}

\subsection{KesA structure}

The three dimensional atomic coordinates of the KcsA channel were taken from the crystallographic structure determined at $2 \AA$ resolution by Y. Zhou et al. [9] (file 1K4C.pdb of the Protein Data Bank [10]). The atomic coordinates of the first 22 amino acids at the $\mathrm{N}$ terminal and of the last 45 at the $\mathrm{C}$ terminal were not experimentally determined. Since both $\mathrm{C}$ and $\mathrm{N}$ terminal are located in the cytoplasm, far from the conduction pathway, these amino acids are not crucial for the present study and therefore, they were not included in the channel model. Side chains with missing atoms were completed using ideal internal coordinate from the AMBER force field [11]. AMBER force field was used to define atomic radii and partial charges too.

The experimentally determined structure of KcsA corresponds to a closed state of the channel. To compute correctly ion fluxes it was necessary to use an open channel structure. Closing of KcsA is due to a bundle between the four inner helixes at the intracellular channel mouth $[3,6]$. A glycine hinge allows the inner helix bending and the consequent channel opening. This mechanism was suggested by the experimental structure of MthK (file 1LNQ.pdb of the Protein Data Bank). MthK is potassium channel from the methanothermbacter thermautotrophic that, differently from KcsA, was crystallized in an open state. Indeed, MthK inner helixes are bent and the selectivity filter is connected to the 
intracellulare space by a wide pore (minimum radius $12 \AA$ ). To compute a structure of KcsA in an open state we minimized the distance between the inner helix of KcsA and MthK by a rigid rotation of the intracellular side of the KcsA inner helixes around the glycine hinges.

KcsA channel comes into contact with the membrane lipid bilayer by the four outer helixes. These helixes have a hydrophobic segment between the amino acids TRP $113(\mathrm{z}=14.5 \AA)$ and TRP $87(\mathrm{z}=-15.5 \AA)$. We used the position of these amino acids to define the thickness of the membrane in our model.

\subsection{Results}

The relative dielectric constant was set to 80 in the water solution and to 2 in the channel and the membrane. Small changes around these values were tested but they did not affect the results significantly. The diffusion coefficients were set to the experimental value, respectively of potassium and chloride $\left(\mathrm{DK}^{+}=1.96 * 10^{-9}\right.$ $\mathrm{m}^{2} / \mathrm{s}, \mathrm{DCl}^{-}=2.03 * 10^{-9} \mathrm{~m}^{2} / \mathrm{s}$ ). Setting the membrane potential to $25 \mathrm{mV}$ and both the extracellular and intracellular ion concentrations to $100 \mathrm{mM}$, the computed current is 4 times the experimental data [12]. The approximation gets worst at higher ion concentration and membrane potential (Fig. 3).

A possible approach to reproduce the experimental data is to use the diffusion coefficients as fitting parameters. Computed currents reproduce quite well the experimental data with a reduction of both the diffusion coefficients to $25 \%$. However, a similar reduction of the diffusion coefficients in the whole water solution is not justifiable. Otherwise, inside the channel, diffusion processes take place differently and it is reasonable to use different values for the diffusion coefficients. We divided the channel in two regions: the hydrophobic pore $(0<\mathrm{z}<-24)$ and the selectivity filter $(14<\mathrm{z}<0)$. The diffusion coefficients were reduced only in these two regions according to the value computed by molecular dynamic simulations (10\% of the experimental value in the selectivity filter, $50 \%$ in the hydrophobic pore) [5]. Using these diffusion coefficients the accordance with the experimental data improves (see Fig. 3).
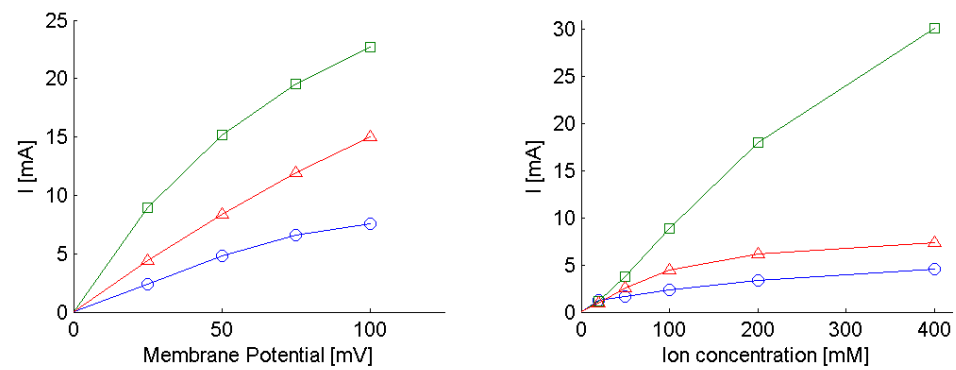

Figure 3: Current-voltage (A) and current-concentration (B) characteristics. Experimental data $(O)$, diffusion coefficients set to the experimental values $(\square)$, reduced diffusion coefficients $(\Delta)$. 


\section{Discussion}

In this paper we have attempted to relate structure and function of ion channels, making use of the KcsA experimental data. To this end we developed a numerical solver of the PNP equations. In ion channel simulation studies, an approach based on the PNP electrodiffusion theory is complementary to an approach based on molecular dynamics. The last can reveal the atomic details about ion channel functioning, while the former, giving up the atomic detail, provide a way to compute ion currents. To simplify the mathematical model of ion conduction, the PNP theory describes the ion distribution with a continuum function. Despite this simplifying assumption the computed currents are in good accordance with the experimental data. It is important to highlight that the accordance between simulated and experimental data was obtained with diffusion coefficient values matching the ones computed by molecular dynamics simulations. Therefore these coefficients must not to be interpreted as fitting parameters. Instead, they connect the microscopic description provided by molecular dynamics to the macroscopic of the PNP theory.

The accordance with the experimental data is good especially with ion concentrations and membrane potential in the physiological range. At higher values an overestimation of the current was obtained, probably due to saturation effects not introduced in the model. Even if an improvement of the mathematical model in this direction is desirable, the present approximation provides a good tool to connect structure and function of ion channels.

\section{Appendix: numerical method}

The numerical procedure used to solve the mass conservation equations (4) is presented here. For sake of simplicity we will refer to a bidimensional case, generalization to the three dimensional case is immediate. The same numerical procedure was used to solve the Poisson's equation (5). The ion flux in the $\mathrm{x}$ direction between the grid elements $(i-1, j)$ and $(i, j)$ was expressed as:

$$
J_{i-1}^{x}=-\frac{D_{i, j}+D_{i-1, j}}{2}\left[\frac{C_{i, j}-C_{i-1, j}}{h}+\frac{z e}{k_{B} T} \frac{C_{i, j}+C_{i-1, j}}{2} \frac{\psi_{i, j}-\psi_{i-1, j}}{h}\right]
$$

that is the lattice version of equation (1) (h is the grid step). The ion fluxes $J_{i+1}^{x}$ - in the x direction between the elements $(\mathrm{i}, \mathrm{j})$ and $(\mathrm{i}+1, \mathrm{j})-, J_{j-1}^{y}$ and $J_{j+1}^{y}$ were expressed consistently. According to the mass conservation law, net steady state flux through any grid element is zero, that is:

$$
J_{i-1}^{x}-J_{i+1}^{x}+J_{j-1}^{y}-J_{j+1}^{y}=0
$$


Replacing in this equation the fluxes expressed as in (A1) it is possible to write:

$$
C_{i, j}=\frac{\sum_{k} C_{k} \frac{D_{i, j}+D_{k}}{2}\left[1-\frac{e z}{2 k_{B} T}\left(\psi_{i, j}-\psi_{k}\right)\right]}{\sum_{k} \frac{D_{i, j}+D_{k}}{2}\left[1+\frac{e z}{2 k_{B} T}\left(\psi_{i, j}-\psi_{k}\right)\right]}
$$

where $\mathrm{k}$ is an index that rounds over the four surrounding grid elements, $\mathrm{k}=(\mathrm{i}$ $1, j),(i+1, j),(i, j-1),(i, j+1)$. Equation (A3) was used in an iterative scheme based on the successive over relaxation techniques [14] to find the solution of the mass conservation equation. At the first step ion concentration in each grid element is set randomly, these concentrations are then updated, according to:

$$
C_{i, j}^{n}=(1-w) C_{i, j}^{n-1}+w C_{i, j}
$$

where $C_{i, j}^{n}$ and $C_{i, j}^{n-1}$ are respectively the concentration computed at the step $\mathrm{n}$ and n-1, and $C_{i, j}$ is obtained by (A3). Setting correctly the weight w, the number of iteration to reach the solutions drops appreciably. To test the convergence to solution the root mean square distance (RMSD) between two successive iterations is used, the procedure is stopped when RMSD falls below $10^{-3}$.

\section{References}

[1] Hille, B., Ionic channels of excitable membranes, 2nd Ed. Sinauer Associates, Sunderland, MA, 1992.

[2] Doyle, D.A., The structure of the potassium channel: molecular basis of $\mathrm{K}+$ conduction and selectivity, Science, 1998, vol. 280, no. 5360, pp. 69-77.

[3] Jiang, Y., Crystal structure and mechanism of a calcium-gated potassium channel, Nature, 2002, vol. 417, no. 6888, pp. 515-22.

[4] Jiang, Y., X-ray structure of a voltage-dependent $\mathrm{K}+$ channel, Nature, 2003, vol.4030, no. 7001,pp. 806-10.

[5] Allen, T.W., The potassium channel: structure, selectivity and diffusion, Biophys J., 1999, vol. 77, no. 5, pp. 2502-16.

[6] Perozo, E., Structural rearrangements underlying K+-channel activation gating, Science, 1999, vol. 285, no. 5424, pp. 73-8.

[7] Bernechè, S., A microscopic view of ion conduction through the $\mathrm{K}+$ channel, PNAS, 2003, vol. 100, no. 15, pp. 8644-48. 
[8] Rocchia, W., Rapid grid-based construction of the molecular surface for both molecules and geometric objects: application to the finite difference Poisson-Boltzmann method, J. Comp. Chem., 2002, vol. 23, pp.128-37.

[9] Zhou, Y., Chemistry of ion coordination and hydration revealed by a K+ channel-Fab complex at $2.0 \AA$ Å resolution, Nature, 2001, vol. 414, no.6859, pp. 44-8.

[10] Protein Data Bank, www.rcsb.org

[11] Pearlman, D.A., AMBER, a package of computer programs for applying molecular mechanics, normal mode analysis, molecular dynamics and free energy calculations to simulate the structural and energetic properties of molecules. Comp. Phys. Commun., 1995, vol. 91, pp.1-41.

[12] LeMasurier, M., KcsA: it's a potassium channel, J Gen Physiol, 2001, vol. 118, no. 3, p. 303-314.

[13] Young, D.M., Iterative solution of large linear systems, 1971, Academic press: New York and London. 